\title{
The clinical and radiological characteristics of pulmonary cryptococcosis in immunocompetent and immunocompromised patients
}

\author{
Yan $\mathrm{Hu}^{1}$, Si-Ying Ren ${ }^{2}$, Peng Xiao ${ }^{3}$, Feng-Lei Yu ${ }^{1}$ and Wen-Liang Liu ${ }^{1 *}$
}

\begin{abstract}
Background: We characterized the clinical features, radiographic characteristics, and response to treatment of immunocompetent and immunocompromised patients with pulmonary cryptococcosis (PC).

Methods: We retrospectively reviewed the medical records and radiological profiles of patients diagnosed with PC who received surgical resection between May 2015 and November 2020 in a tertiary referral center.

Results: A total of 21 males and 18 females were included in the study. 23 patients were immunocompetent and 20 out of the 39 were asymptomatic. Immunocompetent patients were diagnosed with PC at a younger age than immunocompromised patients ( 48.9 vs 57.1 years, $P=0.02$ ). Single nodule pattern was the most frequent lesion pattern (33 out of $39,84.6 \%$ ) and right upper lobe was the most common site of location (15 out of $47,31.9 \%$ ). The majority of lesions were located peripherally (38 out of $47,80.9 \%$ ) and most lesions were $1-2 \mathrm{~cm}$ in diameter (30 out of $47,63.8 \%$ ). Cavitation was more likely to occur in immunocompromised patients (5 out of $11,45.5 \%$ ) than in immunocompetent patients (6 out of $36,16.7 \%)(P=0.04)$ and there was complete resolution of $P C$ in all patients treated with anti-fungal therapy.

Conclusions: Immunocompetent patients were diagnosed with PC at a younger age than immunocompromised patients. Single nodule pattern was the most frequent lesion pattern in PC patients. Cavitation was more likely to occur in immunocompromised patients than in immunocompetent patients.
\end{abstract}

Keywords: Pulmonary cryptococcosis, Computed tomography, Fungal infection, Immune status, Cavitation

\section{Background}

Pulmonary Cryptococcosis (PC) is a pulmonary fungal disease caused by the inhalation of Cryptoccocus neoformans or Cryptococcus gattii spores into the respiratory system [1]. It is well-known that PC mostly occurs in immunocompromised patients, including AIDS patients,

\footnotetext{
*Correspondence: liuwenliang@csu.edu.cn

${ }^{1}$ Department of Thoracic Surgery, The Second Xiangya Hospital

of Central South University, No. 139 Renmin Road, Changsha 410011,

China

Full list of author information is available at the end of the article
}

patients with hematologic malignancies, organ transplantation recipients, and patients receiving immunosuppressive agents $[2,3]$. However, the incidence of PC in immunocompetent patients has increased in recent years $[4,5]$. Radiographically, PC may appear as a solitary nodule or mass, multifocal nodules, pulmonary consolidations, or bronchopneumonia [6, 7]. It is difficult to differentiate PC from primary lung cancer, tuberculosis, or metastasis especially when PC presents as a solitary pulmonary nodule. The aim of this retrospective study is to characterize the clinical features, radiographic 
characteristics, and response to treatment of immunocompetent and immunocompromised patients with PC.

\section{Methods}

\section{Study subjects}

We retrospectively reviewed the medical records and radiological profiles of patients diagnosed with $\mathrm{PC}$ who received surgical resection in our center between May 2015 and November 2020. Patients with PC would be included in the study if they met all of the following conditions: 1 . presence of pulmonary nodule or mass in which lung cancer could not be ruled out clinically; 2. PC was histologically and microbiologically confirmed; 3 . clinical data and CT imaging information were available.

The following clinical data were extracted from the medical records: sex, age, presenting symptoms, cormorbidities, smoking status, surgical and antifungal therapy, pathology, follow-up, and prognostic outcomes. Chest CT images were analyzed and the location, pattern, size, margins, pleural sign, and presence of air bronchogram, cavitation, Halo sign, and mediastinal lymphoadenopathy were recorded. Positron emission tomography (PET)/ CT images were evaluated for standardized uptake values (SUVs) if accessible. Pulmonary Lesions or lymph nodes with higher FDG uptake was defined when their maximum SUV $\left(\mathrm{SUV}_{\max }\right)$ was higher than that in the normal lung tissues or the mediastinal blood pool, respectively. Diagnosis of PC was confirmed by postoperative pathology. All samples were formalin-fixed, paraffin-embedded, and stained with haematoxylin-eosin (H\&E), Gomori methenamine silver (GMS), and periodic acidSchiff (PAS). The Institutional Review Boards of Second Xiangya Hospital approved the study.

\section{Statistical analysis}

Descriptive analyses of patient demographics, clinical features, radiological findings, pathological data, and treatment outcomes were performed. All data were presented as mean $\pm \mathrm{SD}$ for continuous variables, and as numbers with percentages for categorical variables. The Student's test was used for continuous variables and Pearson $X^{2}$ test or Fisher's exact test for categorical variables. All statistical analyses were performed using STATA software. $P<0.05$ was considered to indicate statistical significance.

\section{Results}

\section{Patient demographics and clinical features}

A total of 21 males (53.8\%) and 18 females (46.2\%) were included in the study. The mean age was 51.1 years (standard deviation: 10.9). There were 32 non-smokers and seven current or ex-smokers. Of the 39 patients, 27 were asymptomatic (69.2\%), and presented with an incidental finding of pulmonary lesions following routine health examinations. In the other 12 patients, symptoms including cough $(n=6)$, sputum $(n=6)$, chest pain $(n=4)$, chest distress $(n=3)$, hemoptysis $(n=1)$, and fever $(n=1)$ were observed. In terms of comorbidities, nine patients had hypertension, eight had a malignancy, two had previous environmental exposures (a clear history of exposure to sulfides $(n=1)$ or coal $(n=1))$, one had severe diabetes mellitus, one received bilateral kidney transplantation, and one had a previous history of pulmonary tuberculosis. For host immune status, 29 patients were immunocompetent. The other 10 patients were considered immunocompromised, including eight patients with a malignancy, one with organ transplantation and diabetes mellitus, and one with tuberculosis receiving antituberculosis drugs. Immunocompetent patients were diagnosed with $\mathrm{PC}$ at a younger age than immunocompromised patients (48.9 vs 57.1 years, $P=0.02$ ). None of the patients had AIDS at the time of diagnosis of PC. 38 patients were diagnosed with PC using surgical modality and only one patient was diagnosed using percutaneous translung biopsy before surgery. Type of surgery included wedge resection $(n=22)$, segmentectomy $(n=3)$, and lobectomy $(n=14)$. The initial suspected diagnosis before surgical resection of the lesion(s) was primary lung cancer in 20 lesions, pulmonary metastasis in five lesions, non-specific granulomatous disease in six lesions, pulmonary tuberculosis in two lesions, infectious disease in two lesions, benign lesions in nine lesions, and hamartoma in two lesions. Immunocompromised patients were more likely to obtain initial diagnosis of pulmonary metastasis than immunocompetent patients $(P=0.001)$. Patient demographic and clinical information is summarized in Table 1.

\section{Radiologic presentation findings}

The thoracic CT findings are summarized in Table 2. Single nodule pattern was the most frequent lesion pattern, presenting in 33 of the 39 (84.6\%) patients, followed by scattered nodule $(7.7 \%)$, clustered nodule (5.1\%), and mass-like (2.5\%) (Fig. 1). Pleural effusion was not seen in any patient and mediastinal lymph node enlargement was observed in 10 patients. Most lesions were located in the right upper lobe ( 15 lesions, $31.9 \%$ ), followed by right lower lobe (13 lesions, 27.6\%) and left lower lobe (13 lesions, 27.6\%). Pulmonary lesions (38 out of $47,80.9 \%$ ) were located mostly in the peripheral lung field (outer third of the lung). The diameter of the lung lesions was less than $1 \mathrm{~cm}$ in nine lesions (19.1\%), $1-2 \mathrm{~cm}$ in 30 lesions (63.8\%), and greater than $2 \mathrm{~cm}$ in eight lesions (17\%). 20 (42.6\%) lesions were spiculated. Air bronchogram was noted in three lesions (6.4\%), halo sign in nine (19.1\%), and 
Table 1 Demographics and clinical features of patients with PC

\begin{tabular}{|c|c|c|c|c|}
\hline Clinical characteristics & Total $(n=39)$ & $\begin{array}{l}\text { Immunocompetent } \\
\text { patients }(n=29)\end{array}$ & $\begin{array}{l}\text { Immunocompromised } \\
\text { patients }(n=10)\end{array}$ & $P$ values \\
\hline Gender (male) & 21 & 16 & 5 & 0.78 \\
\hline Current or ex-smoker & 7 & 4 & 3 & 0.25 \\
\hline \multicolumn{5}{|l|}{ Clinical symptoms } \\
\hline Cough & 6 & 4 & 2 & 0.64 \\
\hline Sputum & 6 & 4 & 2 & 0.64 \\
\hline Chest pain & 4 & 2 & 2 & 0.24 \\
\hline Chest distress & 3 & 2 & 1 & 0.75 \\
\hline Hemoptysis & 1 & 1 & 0 & 0.55 \\
\hline Fever & 1 & 0 & 1 & 0.08 \\
\hline \multicolumn{5}{|l|}{ Comorbidities } \\
\hline Malignancy & 8 & 0 & 8 & \\
\hline Environmental exposures & 2 & 2 & 0 & \\
\hline Diabetes mellitus & 2 & 0 & 2 & \\
\hline History of tuberculosis & 1 & 0 & 1 & \\
\hline History of organ transplantation & 1 & 0 & 1 & \\
\hline \multicolumn{5}{|l|}{ Diagnosis by } \\
\hline Surgery & 38 & 29 & 9 & \\
\hline Percutaneous translung biopsy & 1 & 0 & 1 & \\
\hline Type of surgery & & & & 0.19 \\
\hline Wedge resection & 22 & 18 & 4 & \\
\hline Lobectomy & 14 & 10 & 4 & \\
\hline Segmentectomy & 3 & 1 & 2 & \\
\hline \multicolumn{5}{|l|}{ Initial diagnosis before surgery } \\
\hline Primary lung cancer & 20 & 18 & 2 & 0.06 \\
\hline Pulmonary metastasis & 5 & 0 & 5 & 0.001 \\
\hline Non-specific granulomatous disease & 6 & 5 & 1 & 0.68 \\
\hline Pulmonary tuberculosis & 2 & 2 & 0 & 0.42 \\
\hline Infectious disease & 2 & 2 & 0 & 0.42 \\
\hline Benign lesions & 9 & 7 & 2 & 0.93 \\
\hline Pulmonary cryptococcosis & 1 & 0 & 1 & 0.06 \\
\hline Hamartoma & 2 & 2 & 0 & 0.42 \\
\hline
\end{tabular}

pleural indentation in 10 (21.3\%). Cavitation was noted in 11 lesions and was more likely to occur in immunocompromised patients (5 out of $11,45.5 \%$ ) than in immunocompetent patients (6 out of $36,16.7 \%$ ) $(P=0.04)$.

The PET/CT results are summarized in Table 3. A total of 14 patients underwent PET/CT scans. SUV $\max$ ranged from 1.8 to 43.5 (average $9.12 \pm 10.46$, median 6.3). All of the patients showed higher FDG uptake in the pulmonary lesions and four patients revealed higher uptake in lymph node areas. There was no significant difference in $\mathrm{SUV}_{\max }$ between immunocompetent and immunocompromised patients $(P=0.49)$.

\section{Pathological diagnosis}

Diagnosis of PC was confirmed in all patients by postoperative pathology. H\&E staining showed a variety of inflammatory reactions from inflammation to wellformed granulomas, accompanied by various degrees of fibrosis and necrosis (Fig. 2). The presence of the cryptococcal pathogen was identified in all samples by histochemically staining with Gomori methenamine silver (GMS) and periodic acid-Schiff (PAS).

\section{Treatment and prognosis}

The follow-up period for the patients ranged from 2.5 to 48 months (mean, 11.8 months). Of the 38 patients who were diagnosed by surgery, one patient was lost to 
Table 2 Thoracic CT findings of patients with PC

\begin{tabular}{|c|c|c|c|c|}
\hline CT Findings & $\begin{array}{l}\text { Total } \\
\text { patient }(\mathrm{n}=39)\end{array}$ & $\begin{array}{l}\text { Immunocompetent } \\
\text { patients }(\mathrm{n}=29)\end{array}$ & $\begin{array}{l}\text { Immunocompromised } \\
\text { patients }(\mathrm{n}=10)\end{array}$ & $\begin{array}{l}P \\
\text { value }\end{array}$ \\
\hline Lesion pattern & & & & 0.29 \\
\hline Single nodule & 33 & 25 & 8 & \\
\hline Scattered nodule & 3 & 2 & 1 & \\
\hline Clustered nodule & 2 & 2 & 0 & \\
\hline Mass-like & 1 & 0 & 1 & \\
\hline Pleural effusion & 0 & 0 & 0 & \\
\hline Mediastinal lymphoadenopathy & 10 & 6 & 4 & 0.23 \\
\hline Location of the lesions & Total lesions $(n=47)$ & & & 0.06 \\
\hline RUL & 15 & 12 & 3 & \\
\hline RML & 1 & 0 & 1 & \\
\hline RLL & 13 & 12 & 1 & \\
\hline LUL & 5 & 2 & 3 & \\
\hline LLL & 13 & 10 & 3 & \\
\hline Distribution of the lesions & & & & 0.43 \\
\hline Peripheral & 38 & 30 & 8 & \\
\hline Central location & 9 & 6 & 3 & \\
\hline Diameter of the lesions & & & & 0.71 \\
\hline$<1 \mathrm{~cm}$ & 9 & 6 & 3 & \\
\hline $1-2 \mathrm{~cm}$ & 30 & 24 & 6 & \\
\hline$>2 \mathrm{~cm}$ & 8 & 6 & 2 & \\
\hline Spiculation & 20 & 16 & 4 & 0.64 \\
\hline Air bronchogram & 3 & 3 & 0 & 0.32 \\
\hline Cavitation & 11 & 6 & 5 & 0.04 \\
\hline Halo sign & 9 & 7 & 2 & 0.93 \\
\hline Pleural indentation & 10 & 8 & 2 & 0.77 \\
\hline
\end{tabular}

follow up and the remaining 37 patients received postoperative antifungal therapy (fluconazole $400 \mathrm{mg} /$ day). The duration of the antifungal therapy ranged from 2 weeks to 8 months (mean, 3.5 months). One patient obtained the diagnosis of PC before surgery. She received oral fluconazole ( $400 \mathrm{mg} /$ day) for 6 months preoperatively and 3 months postoperatively. All of the 38 patients who received follow-up were completely cured after treatment.

\section{Discussion}

PC is an important opportunistic fungal disease in immunocompromised patients, but it is also increasingly identified in immunocompetent patients [1]. Previous studies have shown that immunocompetent patients were diagnosed with PC at a younger age than immunocompromised patient $[2,3,8]$. This finding was also seen in our study, which showed that immunocompetent patients were eight years younger than immunocompromised patients ( 48.9 vs 57.1 years, $\mathrm{P}=0.02)$. $\mathrm{PC}$ generally presents with a variety of clinical presentations, from asymptomatic to nonspecific symptoms including cough, sputum, and chest pain [9-11]. In the present study, 27 patients were asymptomatic and the other 12 patients presented with a variety of symptoms such as cough $(\mathrm{n}=6)$, sputum $(\mathrm{n}=6)$, chest pain $(\mathrm{n}=4)$, chest distress $(n=3)$, hemoptysis $(n=1)$, and fever $(n=1)$. Therefore, the variability in the clinical presentation of $\mathrm{PC}$ means that it is not possible to confidently confirm the diagnosis based on clinical characteristics alone [12].

The characteristics of chest $\mathrm{CT}$ findings in $\mathrm{PC}$ has been well described in the literature $[13,14]$. The radiological presentations of PC varied from a solitary pulmonary nodule or mass, multiple nodules, lobular, segmental or subsegmental consolidation, to bronchopneumonia [1, 6]. Several studies reported that single nodule pattern $[6,9,13,15]$ and peripheral distribution of lesions $[3,6$, $8,11,16]$ were the most common pulmonary CT findings. These observations were supported by our study, which showed that the single nodule pattern was the most commonly identified radiological pattern (33 out of $39,84.6 \%$ ) and that most lesions (38 out of $47,80.9 \%$ ) were located in the peripheral lung field. PC may tend to involve the lower lobes $[4,9]$, specifically the right lower 


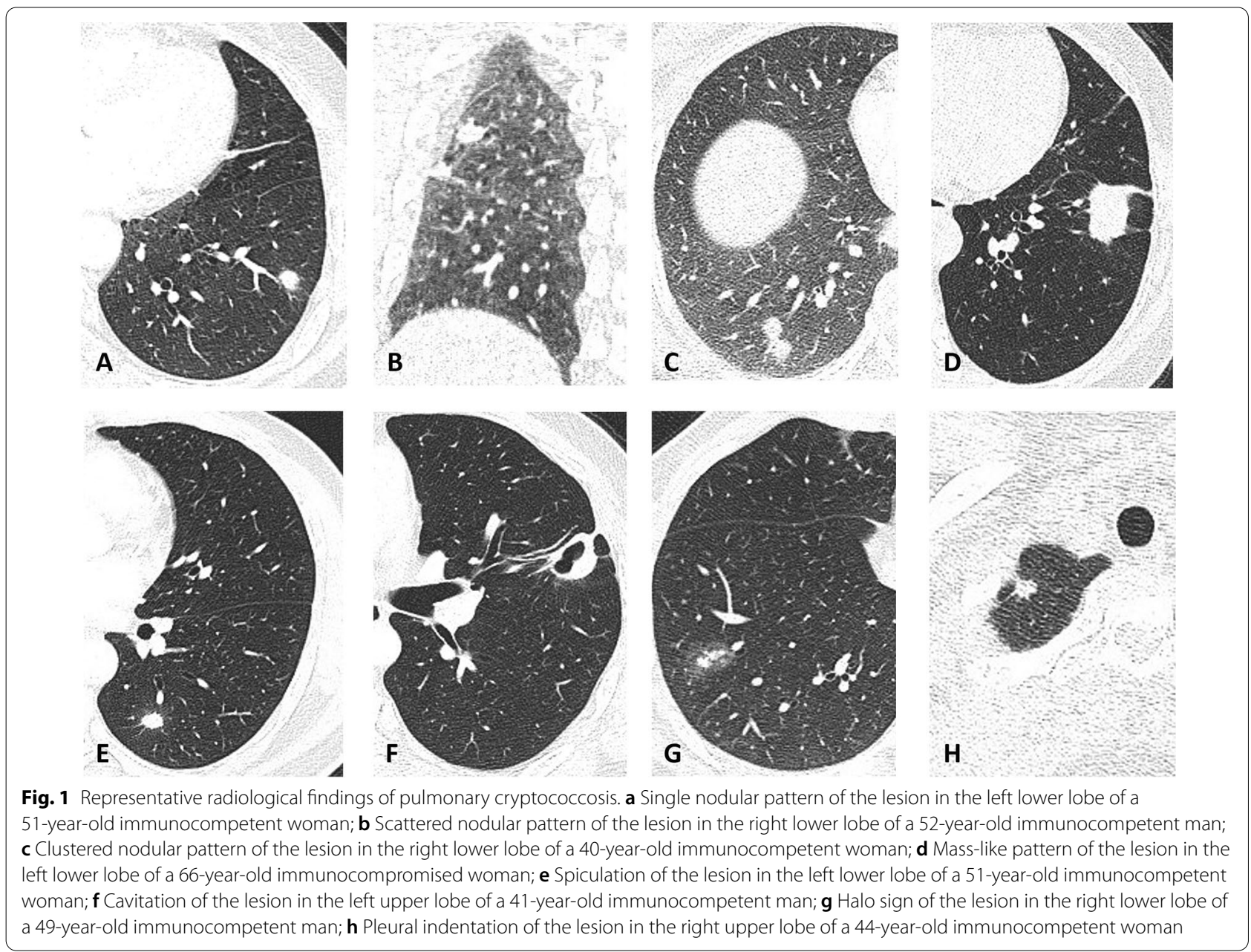

Table 3 PET/CT results of patients with PC

\begin{tabular}{lllll}
\hline PETCT findings & $\begin{array}{l}\text { Total } \\
(\mathbf{n}=\mathbf{1 4})\end{array}$ & $\begin{array}{l}\text { Immunocompetent } \\
\text { patients }(\mathbf{n}=\mathbf{7})\end{array}$ & $\begin{array}{l}\text { Immunocompromised } \\
\text { patients }(\mathbf{n}=\mathbf{7})\end{array}$ & $\begin{array}{l}\boldsymbol{P} \text { value } \\
\text { SUVmax }\end{array}$ \\
Pulmonary lesions with higher uptake & $9.12 \pm 10.46$ & $11.16 \pm 14.58$ & $7.1 \pm 3.85$ \\
Lymph nodes with higher uptake & 14 & 7 & 7 & 0.49 \\
\hline
\end{tabular}

lobe $[6,8,11]$. However, a study by Sui et al. reported that $\mathrm{PC}$ was not associated with any particular lung lobe [13]. In the present study, we found that PC was most commonly located in the right upper lobe, followed by the right lower lobe and left lower lobe.

It is believed that cavitation suggests a long-term focal lung abnormality [2]. Patients with cavitary lesions probably undergo a more severe cryptococcal infection that requires a more aggressive antifungal therapy [2]. Several studies observed that immunocompromised patients were more likely to develop severe pulmonary abnormalities characterized by cavitation than immunocompetent patients $[2,9,13]$. Our study showed similar findings. Higher FDG uptake often occurs in PC lesions $[6,11$, $13,15,17]$. A study by Wang et al. reported that PC lung lesions and mediastinal lymph nodes with increased uptake were seen in 37 and 8 out of 42 PC patients, respectively [6]. In our study, all of the 14 patients who underwent PET/CT showed higher FDG uptake in the pulmonary lesions, and four patients had higher FDG uptake in lymph node areas.

The treatment approach to cryptococcal infection depends on the host immune status, the extent of the disease, the presence of symptoms, and whether the 


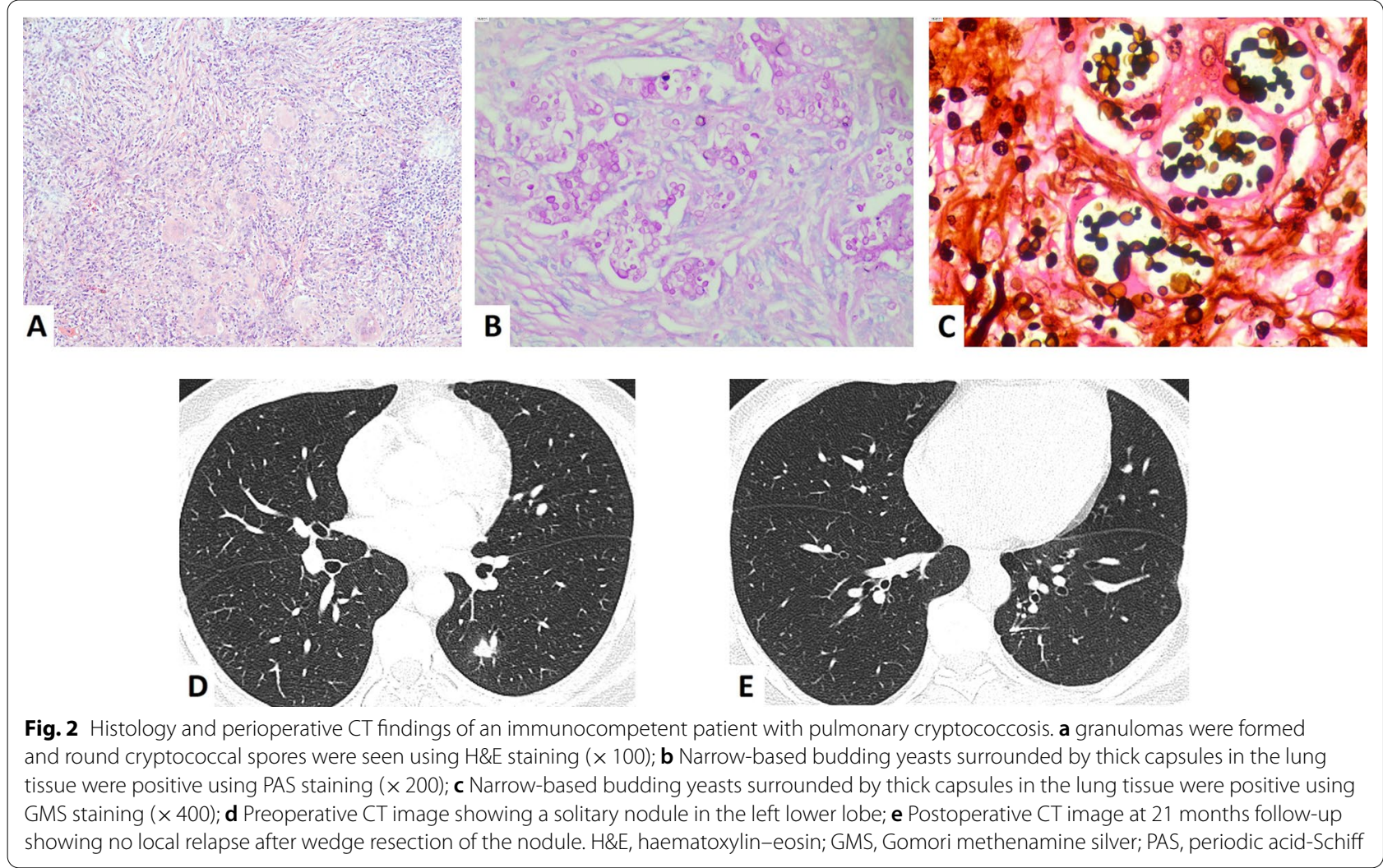

infection is localized to the lungs or central nerve system, or whether it is disseminated [18-20]. Most PC infections in immunocompetent patients are treated with fluconazole, with the addition of amphotericin B in immunocompromised patients, CNS Cryptococcus, or those with diffuse pulmonary disease [21]. However, the management of PC in immunocompetent patients is uncertain [5]. Several previous studies have reported the effectiveness of antifungal therapy alone in the successful treatment of PC $[5,8,22]$. Studies by Nadrous et al. [22] and Zhang et al. [11] reported that simple observation without antifungal therapy may be enough for treating asymptomatic immunocompetent patients with isolated PC, as the lung lesions seen in these patients may undergo spontaneous remission.

Surgical resection is a key component of the modern diagnosis and treatment of fungal lung diseases [5]. Surgery not only affords reliable diagnosis but also represents a treatment modality for many patients with PC, and it may be the optimal management approach for those patients who have undiagnosed isolated PC lesions. Previous studies have shown no relapse of PC after surgery $[6,9,13,15]$. In addition, whether adjuvant antifungal therapy may be required after surgery remains to be determined. Yang et al. suggested no need for additional antifungals by showing that 15 patients underwent surgical resection alone and no patient who underwent surgical resection developed progressive disease [5]. However, this evidence should be considered cautiously because of the limited sample size and short followup durations in their study. In the present study, aside from one patient who was lost to follow-up, all of the other $(n=38)$ patients received postoperative antifungal therapy, and all of these patients showed no evidence of relapse with a mean follow-up of 11.8 months.

There were several limitations to our study. Firstly, this was a single-center retrospective study by design, and therefore may be associated with selection bias. Secondly, immunocompetent and immunocompromised patients were grouped according to their known pre-existing comorbidities/medical history. Immune function testing would be required to definitively establish the immune status of included patients [11]. Thirdly, the number of PC patients included in the study was relatively small. Future large-scale study with longer-term follow-up is warranted.

In conclusion, immunocompetent patients were diagnosed with PC at a younger age than immunocompromised patients. Single nodule pattern was the most frequent lesion pattern in PC patients. Cavitation was more likely to occur in immunocompromised patients than in immunocompetent patients. 


\section{Abbreviations}

PC: Pulmonary cryptococcosis; PET: Positron emission tomography; SUVs: Standardized uptake values.

\section{Acknowledgements}

Not applicable

\section{Authors' contributions}

$\mathrm{YH}$ and WL conceived the project. YH and SR collected and interpreted the data and preprared the figures and tables. PX and FY gave advice on statistical analysis. YH prepared the manuscript with input from all other authors. WL performed the final check of the manuscript. All authors read and approved the final manuscript.

\section{Funding}

This work was supported by the National Natural Science Foundation of China (81972638 and 81700070), the Hunan Province Natural Science Foundation of China (2018JJ3777 and 2019JJ30038), and the Hunan Province Health Department Research Fund (B2018-0541).

\section{Availability of data and materials}

The data that support the findings of this study are available from the corresponding author upon reasonable request.

\section{Declarations}

\section{Ethics approval and consent to participate}

The study was approved by the Clinical Research Ethics Committee of the Second Xiangya Hospital, Central South University (No. 2021-ERCR001) and individual informed consent for this retrospective analysis was waived. This study was performed in accordance with the 1964 Declaration of Helsinki.

\section{Consent for publication}

Not applicable.

\section{Competing interests}

The authors declare that they have no competing interests.

\section{Author details}

'Department of Thoracic Surgery, The Second Xiangya Hospital of Central South University, No. 139 Renmin Road, Changsha 410011, China. ${ }^{2}$ Department of Respiratory and Critical Care Medicine, The Second Xiangya Hospital of Central South University, Changsha 410011, China. ${ }^{3}$ Department of Cardiothoracic Surgery, The Third Xiangya Hospital of Central South University, Changsha 410013, China

Received: 26 February 2021 Accepted: 3 August 2021

Published online: 13 August 2021

\section{References}

1. Chang CC, Sorrell TC, Chen SC. Pulmonary Cryptococcosis. Semin Respirat Crit Care Med. 2015;36(5):681-91.

2. Chang WC, Tzao C, Hsu HH, Lee SC, Huang KL, Tung HJ, et al. Pulmonary cryptococcosis: comparison of clinical and radiographic characteristics in immunocompetent and immunocompromised patients. Chest. 2006;129(2):333-40.

3. Liu K, Ding H, Xu B, You R, Xing Z, Chen J, et al. Clinical analysis of nonAIDS patients pathologically diagnosed with pulmonary cryptococcosis. J Thorac Dis. 2016;8(10):2813-21.

4. Ye F, Xie JX, Zeng QS, Chen GQ, Zhong SQ, Zhong NS. Retrospective analysis of $76 \mathrm{immunocompetent} \mathrm{patients} \mathrm{with} \mathrm{primary} \mathrm{pulmonary}$ cryptococcosis. Lung. 2012;190(3):339-46.
5. Yang B, Lee H, Lee T, Kim SH, Kim MY, Jeong BH, et al. The use of surgery in a real-world clinic to diagnose and treat pulmonary cryptococcosis in immunocompetent patients. J Thorac Dis. 2019;11(4):1251-60.

6. Wang SY, Chen G, Luo DL, Shao D, Liu ET, Sun T, et al. (18)F-FDG PET/CT and contrast-enhanced CT findings of pulmonary cryptococcosis. Eur J Radiol. 2017;89:140-8.

7. Hu Z, Chen J, Wang J, Xiong Q, Zhong Y, Yang Y, et al. Radiological characteristics of pulmonary cryptococcosis in HIV-infected patients. PLoS ONE. 2017:12(3):e0173858.

8. Kohno S, Kakeya H, Izumikawa K, Miyazaki T, Yamamoto Y, Yanagihara $\mathrm{K}$, et al. Clinical features of pulmonary cryptococcosis in non-HIV patients in Japan. J Infect Chemotherapy: Off J Jpn Soc Chemotherapy. 2015;21(1):23-30

9. Kishi K, Homma S, Kurosaki A, Kohno T, Motoi N, Yoshimura K. Clinical features and high-resolution CT findings of pulmonary cryptococcosis in non-AIDS patients. Respir Med. 2006;100(5):807-12.

10. Guimaraes MD, Marchiori E, Meirelles GS, Hochhegger B, Santana PR, Gross $J$, et al. Fungal infection mimicking pulmonary malignancy: clinical and radiological characteristics. Lung. 2013;191(6):655-62.

11. Zhang Y, Li N, Zhang Y, Li H, Chen X, Wang S, et al. Clinical analysis of 76 patients pathologically diagnosed with pulmonary cryptococcosis. Eur Respir J. 2012;40(5):1191-200.

12. Taniwaki M, Yamasaki M, Ishikawa N, Kawamoto K, Hattori N. Pulmonary cryptococcosis mimicking lung cancer. Lancet Infect Dis. 2019;19(9):1033.

13. Sui $X$, Huang $Y$, Song W, Zheng F, Wang $X$, Xu X, et al. Clinical features of pulmonary cryptococcosis in thin-section CT in immunocompetent and non-AIDS immunocompromised patients. Radiol Med (Torino). 2020;125(1):31-8

14. Murayama S, Sakai S, Soeda H, Yabuuchi H, Masuda K, Inoue H, et al. Pulmonary cryptococcosis in immunocompetent patients: HRCT characteristics. Clin Imaging. 2004;28(3):191-5.

15. Igai $\mathrm{H}$, Gotoh $M$, Yokomise H. Computed tomography (CT) and positron emission tomography with [18F]fluoro-2-deoxy-D-glucose (FDG-PET) images of pulmonary cryptococcosis mimicking lung cancer. Eur J Cardio-Thorac Surg: Off J Eur Assoc Cardio-Thorac Surg. 2006;30(6):837-9.

16. Qu Y, Liu G, Ghimire P, Liao M, Shi H, Yang G, et al. Primary pulmonary cryptococcosis: evaluation of CT characteristics in 26 immunocompetent Chinese patients. Acta Radiol. 2012;53(6):668-74.

17. Huang CJ, You DL, Lee PI, Hsu LH, Liu CC, Shih CS, et al. Characteristics of integrated 18F-FDG PET/CT in Pulmonary Cryptococcosis. Acta Radiol. 2009;50(4):374-8

18. Perfect JR, Dismukes WE, Dromer F, Goldman DL, Graybill JR, Hamill RJ, et al. Clinical practice guidelines for the management of cryptococcal disease: 2010 update by the infectious diseases Society of America. Clin Infect Diseases: Off Publ Infect Diseases Soc Am. 2010;50(3):291-322.

19. Limper AH, Knox KS, Sarosi GA, Ampel NM, Bennett JE, Catanzaro A, et al. An official American Thoracic Society statement: treatment of fungal infections in adult pulmonary and critical care patients. Am J Respir Crit Care Med. 2011;183(1):96-128.

20. Saag MS, Graybill RJ, Larsen RA, Pappas PG, Perfect JR, Powderly WG, et al. Practice guidelines for the management of cryptococcal disease. Infectious Diseases Society of America. Clin Infect Diseases: Off Publ Infect Diseases Soc Am. 2000;30(4):710-8.

21. Beamer S. Surgical management of non-mycobacterial fungal infections. J Thorac Dis. 2018;10(Suppl 28):S3398-407.

22. Nadrous HF, Antonios VS, Terrell CL, Ryu JH. Pulmonary cryptococcosis in nonimmunocompromised patients. Chest. 2003;124(6):2143-7.

\section{Publisher's Note}

Springer Nature remains neutral with regard to jurisdictional claims in published maps and institutional affiliations. 